\title{
CARACTERIZAÇÃO PETROFÍSICA DE RESERVATÓRIO CARBONÁTICO FRATURADO DO GRUPO MACAÉ, BACIA DE CAMPOS
}

\author{
Leandro H. Melani ${ }^{1 *}$, Alexandre C. Vidal ${ }^{1}$ e Frederico B. Schuab ${ }^{1,2}$ \\ ${ }^{1}$ Universidade Estadual de Campinas e ${ }^{2}$ PETROBRAS - Petróleo Brasileiro S.A
}

Copyright 2014, SBGf - Sociedade Brasileira de Geofísica

Este texto foi preparado para a apresentação no VI Simpósio Brasileiro de Geofísica, Porto Alegre, 14 a 16 de outubro de 2014. Seu conteúdo foi revisado pelo Comitê Técnico do VI SimBGf, mas não necessariamente representa a opinião da SBGf ou de seus associados. E proibida a reprodução total ou parcial deste material para propósitos comerciais sem prévia autorização da SBGt.

\section{Resumo}

A bacia de Campos, localizada na região sudeste do Brasil ao longo da costa norte do estado do Rio de Janeiro, é a bacia offshore mais produtiva do Brasil. Reservatórios carbonáticos fraturados do Albiano provaram conter importantes reservas de hidrocarbonetos. A caracterização petrofísica de reservatório visa diminuir o grau de incerteza e os riscos associados à exploração de petróleo e gás, sendo importante na cons trução dos modelos estático e dinâmico do campo, essenciais para obtenção do volume de óleo in place (VOIP). As relações petrofísicas em reservatórios carbonáticos podem ser muito mais complexas em relação à siliciclásticos, devido maior a heterogeneidade na distribuição de fácies e porosidade, acarretando desafios significativos para avaliação petrofísica. A definição de um fluxo de trabalho adequado é muito importante para a caracterização de reservatório, pois os parâmetros petrofísicos estimados devem ser obtidos de modo a garantir o mínimo de erro possível, já que serão utilizados para popular o modelo geológico a fim de se avaliar o potencial econômico do campo.

\section{Introdução}

O grande interesse em carbonatos justifica-se pelo fato de que cerca de $50 \%$ do petróleo no mundo é produzido nesse tipo de rocha. Reservatórios carbonáticos fraturados do Albiano, localizados ao longo do leste bacias marginais brasileiras, provaram conter importantes reservas de hidrocarbonetos. Devido à maior heterogeneidade na distribuição de fácies e porosidade, a caracterização de reservatórios carbonáticos torna-se mais complexa em relação a reservatórios siliciclásticos. Estas heterogeneidades acarretam desafios significativos para avaliação petrofísica, podendo ocasionar elevada incerteza na determinação de parâmetros petrofísicos. Além disso, em alguns campos Albianos as fraturas têm um papel importante na produção.

A descoberta de uma jazida de hidrocarbonetos, assim como de outros bens minerais, compreende uma atividade de risco elevado devido ao número de fatores associados, contribuindo para elevação do grau de incerteza. A avaliação de reservatórios de petróleo torna-se essencial na indústria de petróleo, especialmente para a identificação e avaliação do potencial econômico dos reservatórios.

A caracterização petrofísica de reservatório visa diminuir o grau de incerteza e os riscos associados à exploração de petróleo e gás, sendo importante na construção dos modelos es tático e dinâmico do campo. A necessidade de estimar com maior precisão os parâmetros petrofísicos é importante, pois incertezas nesses parâmetros podem conduzir a erros significativos nos valores de volume de óleo in place (VOIP).

O trabalho de caracterização de um reservatório compreende a geração de curvas interpretadas de porosidade, saturação de água, permeabilidade, eletrofácies etc., obtidas a partir da análise de perfis geofísicos (GR, DT, NPHI, RHOB, ILD), calibrados com dados petrofísicos de laboratório (correlação rochaperfil).

O objetivo deste trabalho é apresentar o fluxo de trabalho desenvolvido para caracterização petrofísica de um reservatório carbonático fraturado Albiano do Grupo Macaé, Bacia de Campos, Brasil. Os parâmetros petrofísicos obtidos serão utilizados na determinação do volume de hidrocarbonetos in place, objetivo principal de um programa de avaliação de formação.

\section{Características Geológicas}

A Bacia de Campos está localizada no sudeste do Brasil, principalmente offshore nos estados do Rio de Janeiro e Espírito Santo, ocupando uma área de $115.000 \mathrm{~km} 2$. Os reservatórios da Bacia de Campos são responsáveis por cerca de $85 \%$ da produção brasileira e representa $90 \%$ do total de reservas brasileiras (Bruhn, 2003). É uma das doze bacias marginais brasileiras que se encontram sob a planície costeira, plataforma continental e talude da porção ocidental do Oceano Atlântico Sul. A sequência estratigráfica da Bacia de Campos inclui as Formações Campos, Macaé e Lagoa Feia (Guardado et al., 2000). A instalação do ambiente marinho começou com a deposição carbonática em condições de águas rasas, seguidos dos siliciclásticos do Grupo de Macaé.

Os reservatórios carbonáticos Albianos de águas rasas $(100-200 \mathrm{~m})$ fazem parte do trend NE, sendo compostos principalmente de grainstones e packstones contendo oncólitos, pelóides, oólitos e raros bioclastos (Spadini et al., 1988). Os melhores reservatórios ocorrem nas fácies oolíticas e grainstones e nos carbonatos de granulação fina. As acumulações de óleo tem um forte controle estrutural por falhas e dobras. A produção de óleo foi estabelecida a partir dos carbonatos Albianos do Grupo Macaé. Os reservatórios carbonáticos do Macaé são formados principalmente por carbonatos de granulação fina depositados em um ambiente de baixa energia. Isto dá uma característica de baixa permeabilidade para a rocha, apesar das elevadas porosidades (20-30\%). A boa produtividade apresentada por alguns poços se deve à presença de fraturas. 


\section{Dados e Métodos}

Para realização do trabalho foram utilizados dados de perfilagem de 18 poços, que compreendem registros de perfilagem de Raios Gama (GR), Sônico (DT), Resistividade (ILD), Densidade (RHOB) e Neutrão (NPHI). Medidas de laboratório (dados de rocha) de porosidade e permeabilidade estão disponíveis para apenas quatro poços. A base de dados foi disponibilizada pela Agência Nacional do Petróleo (ANP). Para a análise e interpretação dos dados foi utilizado software de petrofísica adequado.

O perfil é o registro contínuo de um poço em relação à profundidade, que tem por objetivo obter as propriedades físicas das rochas sem necessidade de amostrá-las durante a perfuração dos poços (Figura 1). Os perfis respondem a diferentes propriedades físicas da rocha, tais como radioatividade, resistividade e densidade, e correspondem a assinaturas de uma rocha.

- Perfil GR detecta a radioatividade natural das rochas, sendo utilizado na identificação de litologia, de minerais radioativos e para o cálculo do volume de argila.

- Perfil DT mede a diferença nos tempos de trânsito de uma onda mecânica através das rochas, o que permite inferir o grau de compactação das rochas, detectar fraturas e estimar a porosidade.

- Perfil ILD fornece a leitura aproximada da resistividade da rocha (Rt), sendo utilizado principalmente para a avaliação da presença ou não de hidrocarbonetos nas rochas.

- Perfil NPHI representa a quantidade de hidrogênio da formação, sendo a resposta função direta da porosidade, pois quase todo o hidrogênio nas formações geológicas ocorre devido à presença de água e/ou hidrocarbonetos nos poros das rochas,

- Perfil RHOB mede a densidade média global da formação (conjunto rocha-fluido), possibilitando determinar a densidade das formações, identificar zonas de gás e estimar a porosidade da rocha.

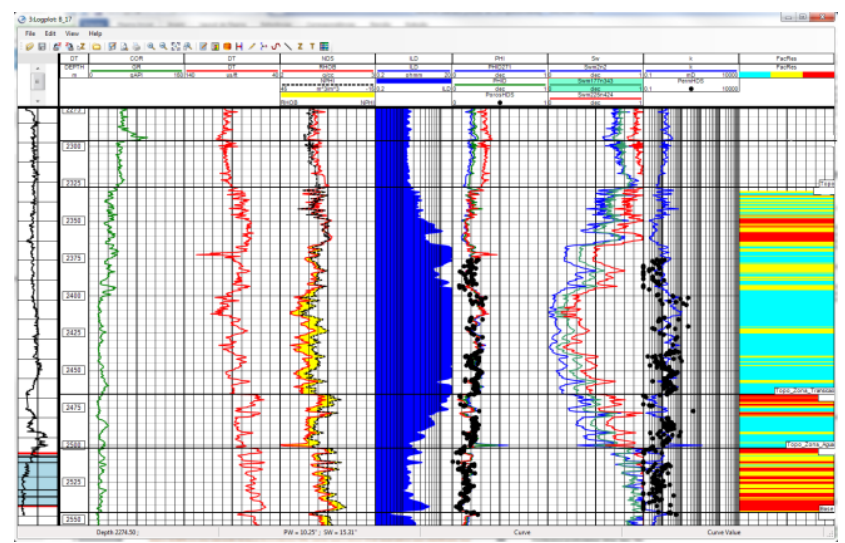

Figura 1 - Exemplo das curvas de perfis geofísicos adquiridos em poços.

A análise petrofísica bas eia-se nas respostas dos perfis elétricos, aliados as informações de crossplots e histogramas que podem fornecer importantes subsídios para o estudo, como diagnósticos ou indicadores da presença de reservatórios, da qualidade desses reservatórios, da composição da matriz dos reservatórios, do tipo litológico e estimativa de porosidade/permeabilidade, da identificação da presença e saturações dos fluidos presentes na rocha.
O fluxo de trabalho (workflow) utilizado neste estudo para obtenção dos parâmetros petrofísicos foi: a) Criação do banco de dados, com o carregamento dos dados de perfilagem e rocha. Não foram efetuadas correções ambientais, por falta de dados adequados. b) Identificação da zona reservatório. c) Cálculo de três curvas de porosidade (PHI) baseada nos perfis sônico (PHIS) e densidade (PHID271 e PHID). d) Calibração dos dados de rocha (rocha-perfil). e) Cálculo da saturação de água $\left(S_{w}\right)$ pela equação de Archie. Também foram construídos três cenários de incerteza para a curva de $S_{\mathrm{w}}$, variando os valores dos parâmetros $m$ e $n$ de Archie. f) Determinação do contato óleo-água, a partir do gráfico Profundidade $x$ $S_{w}$ g) Classificação de eletrofácies pelo método de redes neurais. $h$ ) Construção da curva permeabilidade (k) pelo método da regressão linear. i) Análise de cortes e totalização dos resultados.

\section{Resultados}

A análise petrofísica realizada visou a identificação de potenciais zonas-reservatório no intervalo estudado. Para tanto, foi realizada a delimitação dos intervalos com melhores condições permo-porosas do Grupo Macaé, levando-se em conta os perfis elétricos disponíveis.

\section{- Identificação da zona reservatório:}

Foram delimitados topo e base do reservatório e definidas as zonas de reservatório. O topo foi delimitado analisando-se o comportamento das curvas de perfis, enquanto a base foi marcada no contato óleo-água (OWC), a partir do gráfico Profundidade $x S_{w}$ (Figura 2). Com estes intervalos definidos, foi possível calcular os valores de porosidade e saturação para cada poço. A partir disso, foram subdividas três zonas com base na qualidade de reservatório, sendo elas: zona de óleo, de transição e de água, comum a esses campos Albianos. O volume de hidrocarbonetos é calculado acima do contato óleo-água. Devido a complexidade geológica das rochas carbonáticas as curvas dos perfis apresentam certa variação, dificultando a caracterização dos marcadores de poço.

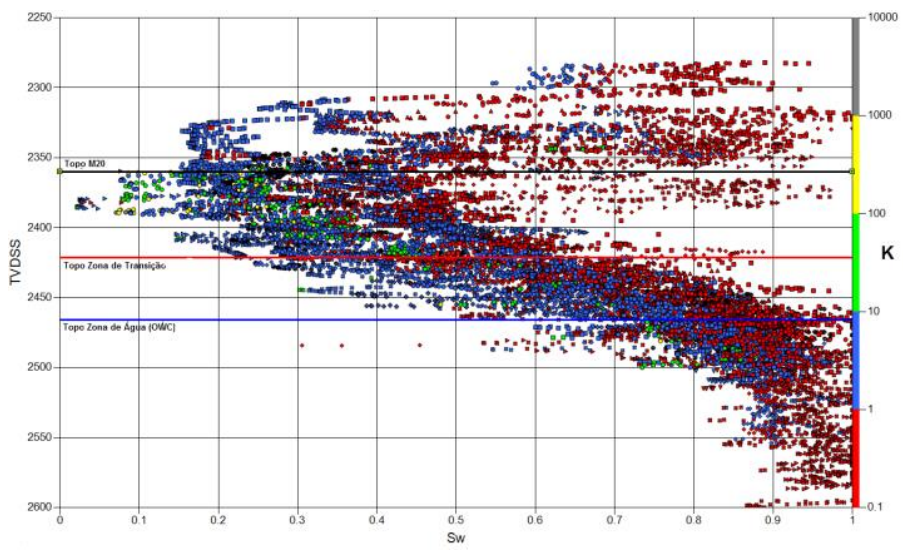

Figura 2 - Gráfico Profundidade $x S_{w}$ auxilia na definição do contato óleo-água (OWC).

\section{- Cálculo da Porosidade (PHI)}

Inicialmente foi calculado o valor de densidade de matriz variável (RHOMAND) para cada poço, a partir 
do crossplot Neutrão (NPHI) x Densidade (RHOB). O crossplot de porosidade N-D auxilia na identificação, de acordo com as linhas de matriz e a distribuição dos pontos no gráfico, do tipo de matriz da rocha (calcítica, dolomítica e arenítica).

Determinada a densidade de matriz variável, foram geradas três curvas de porosidade bas eadas nos perfis sônico (PHIS) e densidade (PHID271 e PHID). A porosidade sônica (PHIS) foi calculada através da equação de Wyllie. As curvas PHID271 e PHID foram calculadas com densidade de matriz fixa $(2,71 \mathrm{~g} / \mathrm{cm} 3$ para calcita) e variável (crossplot N-D), respectivamente, através da equação PHIDen = (RhoMa-RHOB)/(RhoMa-RhoF), onde RhoMa é a densidade da matrize RhoF a densidade do fluído. Os cálculos para estimar a porosidade desconsideram o volume de argila (Vsh $<15 \%$ ), portanto a porosidade total $(\varphi t)$ calculada representa a porosidade efetiva $(\varphi e)$. Hilchie (1978) sugere que o impacto de argila só é significante quando os volumes de argila são maiores que $15 \%$.

As curvas apresentam comportamento semelhante, entretanto a análise das curvas indicou maior correlação rocha-perfil para a curva PHID. Foram observadas variações de até $21,5 \%$ entre as médias das curvas de porosidade quando comparadas entre elas. A porosidade média calculada para a zona de interesse foi de $21,35 \%$.

\section{- Cálculo da saturação de água $\left(S_{w}\right)$}

Para o cálculo de saturação foi aplicado o modelo de Archie, devido os volumes de argila encontrados nos níveis reservatórios não excederem 15\%. Também foram construídos três cenários de incerteza para a curva de $S_{w}$, variando os valores dos parâmetros de cimentação $(m)$ e saturação $(n)$ de Archie. O impacto dessa variação está associado, principalmente, a presença de fraturas e a molhabilidade da rocha. Valores de $m$ inferiores a 2 podem estar relacionados a reservatórios fraturados, enquanto altos valores de $n$ estão relacionados a molhabilidade a óleo das rochas. Valores constantes foram assumidos para o coeficiente tortuosidade "a" $(a=1)$ e para a resistividade da água de formação ( $R w=0,037$ ohm.m), caracterizada através do uso do Pickett Plot (Figura 3).

Para realizar essa verificação, foram comparados os valores de $S_{w} 1,2$ e 3 . No cenário considerado "otimista" $\left(\mathrm{S}_{\mathrm{w}} 1\right)$ foram utilizados os valores clás sicos da equação de Archie $(m=n=2)$, apresentando média de saturação em torno de $35 \%$. O cenário considerado "realista" (C2),apresentou média em torno de 49\%, utilizando $m=1,77$ (gerado a partir do pickett plot) e $n=3,43$ (valor obtido em laboratório para um campo carbonático Albiano análogo da Bacia de Campos). Por fim, no cenário "pessimista" (C3) foi obtida uma média de $61,5 \%$, com $m=2,25$ e $n=4,24$ (valores máximos medidos em laboratório em campo carbonático análogo citado anteriormente). Os resultados mostram variação considerável nos valores médios de $\mathrm{S}_{\mathrm{w}}$, considerandose apenas as zona de óleo e transição. No cálculo da espessura porosa com óleo (H. $\varphi$.So), considerando a zona de óleo do poço B_1, ocorre uma redução de $17 \%$ do cenário realista para o pessimista e um aumento de $15 \%$ do realis ta para o otimista. Os valores de $\mathrm{m}$ e $\mathrm{n}$ para o cenário realista resultaram em uma redução máxima de $19 \%$ no valor de (H.ழ.So) comparado ao otimista no campo B 29. Isso mostra que valores incorretos para os parâmetros de Archie podem subestimar ou superestimar reservas, e assim descaracterizar uma avaliação de viabilidade econômica do campo.

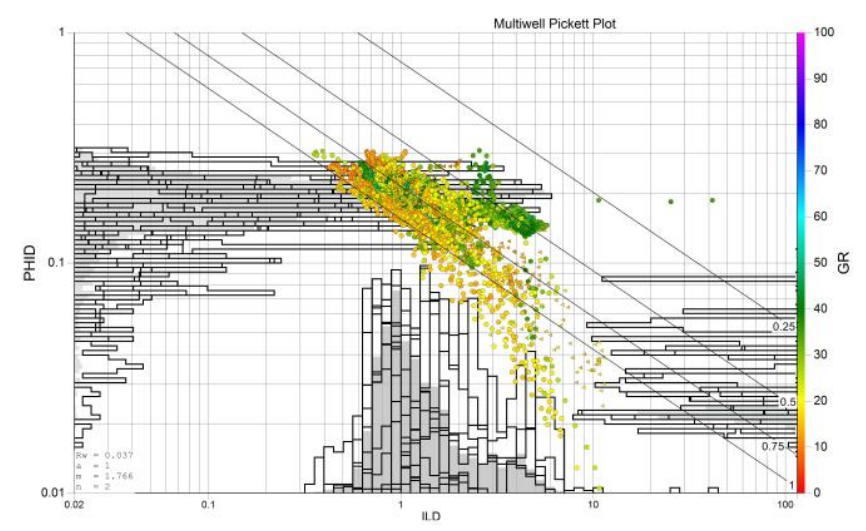

Figura 3 - Pickett Plot para o ajuste de $R w$

\section{- Classificação de Eletrofácies}

Com o objetivo minimizar os problemas referentes a heterogeneidade do campo, a zona reservatório foi dividida em unidades com propriedades petrofísicas similares. Foram geradas as fácies reservatório, possível reservatório e não-reservatório, através do método de redes neurais, baseado no comportamento das curvas dos perfis (Figura 4).

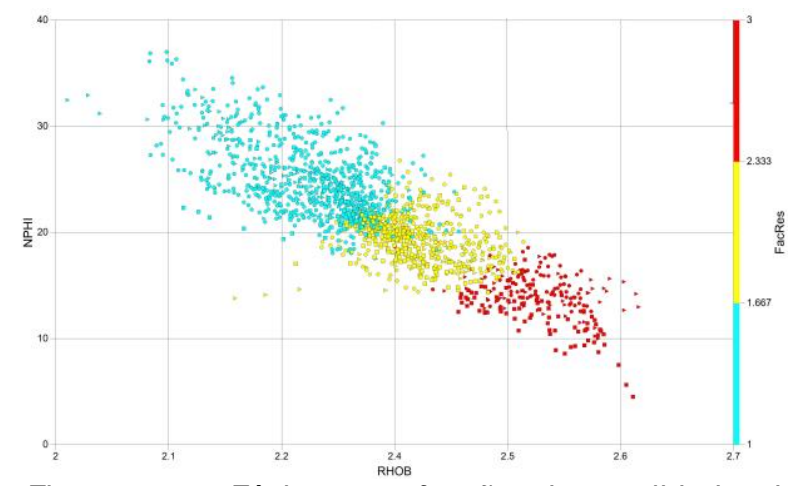

Figura 4 - Fácies em função da qualidade de reservatório, baseadas nos perfis geofísicos.

\section{- Construção da curva permeabilidade (k)}

A construção da curva de permeabilidade foi através do método da regressão linear, baseada na correlação entre porosidade e permeabilidade de plugue. Devido à heterogeneidade das carbonáticas da formação, o resultado das amostras de plugue para permeabilidade apresenta dois comportamentos diferentes de acordo com a posição geográfica dos poços no campo, o que impede o uso de uma mesma abordagem para o reservatório como um todo (Figura 5). Para obtenção de uma curva mais refinada para permeabilidade, foram geradas regressões com amostras agrupadas para cada uma das eletrofácies. Os resultados obtidos mostram em geral uma baixa permeabilidade, apesar de boa porosidade, condizendo com muitos resultados encontrados em reservatórios carbonáticos. A 
permeabilidade média para a zona de interesse foi de $1,591 \mathrm{mD}$, enquanto que para o poço B_30 foi de $60,385 \mathrm{mD}$.

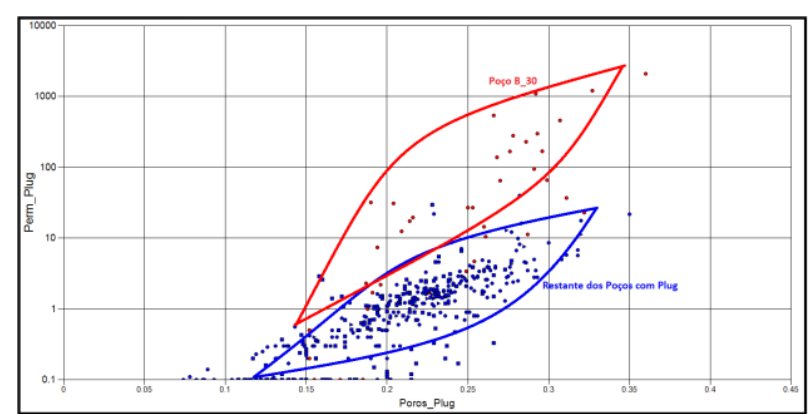

Figura 5 - Diferentes comportamentos de permeabilidade dentro do campo.

\section{- Análise de cortes e totalização dos resultados}

Nesta última etapa foram definidos os parâmetros de corte para o reservatório, o qual é necessário para o cálculo do VOIP.

A partir da observação de alguns gráficos, foi definido que a porosidade mínima de produção é de $14 \%$. Infelizmente, sem a disponibilidade de testemunhos não foi possível afirmar com grau de confiança um valor de corte para saturação de água. A definição de valores de cortes para carbonatos Albianos não é uma tarefa fácil.

Após a definição do corte, os dados foram totalizados, com o intuito de exibir valores que expressam o potencial do reservatório em estudo. Alguns dos resultados obtidos pela totalização serão apresentados em forma de tabelas para cada nível reservatório (Tabela 1).

\begin{tabular}{|c|c|c|}
\hline & Média de PhiD & Média de $\mathrm{S}_{\mathrm{w}}$ \\
\hline Zona Oleo & 0,2152 & 0,4221 \\
\hline Zona Transição & 0,2069 & 0,6201 \\
\hline Zona Agua & 0,1902 & 0,8521 \\
\hline
\end{tabular}

Tabela 1 - Resultados obtidos pela totalização para porosidade e saturação de água em cada zona do reservatório.

\section{Discussão}

A geração de um modelo geológico consistente que honre os dados de produção de reservatórios carbonáticos Albianos não é um trabalho fácil, sendo critica a avaliação de incertezas geológicas para estes reservatórios. A determinação das propriedades petrofísicas é uma das principais fontes de incerteza associada à estimativa do VOIP, um dos principais objetivos da caracterização de reservatórios. Portanto, incertezas nas curvas de porosidade e saturação de água, construídas a partir de dados de perfis, podem afetar significativamente a estimativa volumétrica, confirmando a importância de uma boa caracterização petrofísica para prever reservas com maior precisão, principalmente em reservatórios carbonáticos fraturados.
Quanto a saturação de água, a maior precisão da curva recai na incerteza dos parâmetros de usados na equação de saturação de Archie, que podem acarretar erros não aceitáveis para os valores de saturação de água. Os resultados confirmam a importância dos coeficientes $\mathrm{m}$ e $\mathrm{n}$ para o cálculo de uma curva de saturação de água confiável e na determinação do contato óleo-água e/ou zona de transição, essenciais na redução de incertezas para avaliação de reservas.

Essas informações são importantes para a estimativa de volume e reserva dos reservatórios e tomada de decisão sobre o potencial econômico e aproveitamento do campo.

\section{Agradecimentos}

Aos autores do trabalho Alexandre Campane Viadal e Frederico Bastos Schuab pelo auxílio e orientação técnica.

\section{Referências}

Archie, G. E., 1942. The Electrical Resistivity Log as an Aid in Determining Some Reservoir Characteristics: Journal of Petroleum Technology, v. 5, p. 54-62.

Asquith, G. \& Krygowski, D., 2004. Basic Well Log Analysis: AAPG Methods in Exploration 16. The American Association of Petroleum Geologists. Oklahoma.

Blauth, M., Faria, R.T., Maul, A.R., Monteiro, M.C., Pittella, M., Carneiro, S.R.R., Tibana, P., 2011. Workflow for Geological Characterization and Modeling of the Albian Carbonate Reservoirs from Offshore Campos Basin, Brazil. AAPG International Conference and Exhibition, Milan, Italy. $16 \mathrm{p}$.

Bruhn, C.H.L., Gomes, J.A.T., Del Lucchese Jr., C., Johann, P.R.S., 2003. Campos Basin: Reservoir Characterization and Management - Historical Overview and Future Challenges. Offshore Technology Conference. Houston, Texas, U.S.A. OTC 15220. pp. 1-14.

Glover, P., 2009. What is the cementation exponent? A new interpretation. The Leading Edge, Special Section: Rock Physics. pp.82-85.

Gunter, G.W., Finneran, J.M., Hartmann, D.J., Miller, J.D., 1997. Early Determination of Reservoir Flow Units Using an Integrated Petrophysical Method. SPE Annual Technical Conference and Exhibition, SPE-38679-MS, San Antonio, Texas. 8p.

Guardado, L. R., Spadini, A. R., Brandao, J. S. L., Mello, M. R., 2000. Petroleum System of the Campos Basin, Brazil. In Mello, M.R. and Katz, B.J., eds, Petroleum System of South Atlantic Margins: AAPG Memoir 73, p. 317-324.

Rangel, H. D., Martins, F. A. L., Esteves, F. R., Feijó, F. J., 1994. Bacia de Campos. Boletim de Geociências da Petrobrás, vol. 8. 
